\title{
结合边缘轮廓和姿态特征的人体精确解析模型
}

\author{
甘霖 1$)$ ，刘骊 1,2$)^{*}$ ，刘利军 ${ }^{1,2)}$ ，付晓东 ${ }^{1,2)}$, 黄青松 1,2 ) \\ ${ }^{1)}$ (昆明理工大学信息工程与自动化学院 昆明 650500) \\ 2) (云南省计算机技术应用重点实验室 昆明 650500) \\ (kmust_mary@163.com)
}

\begin{abstract}
摘 要: 针对着装场景中由于人体姿态、边缘轮廓、服装配饰的复杂性以及着装部位关节点被遮挡等因素导致人体 解析精度较低的问题, 提出一种结合边缘轮廓和姿态特征的人体精确解析模型. 首先采用残差网络 ResNet-101 作为 主干网络表征输人人体图像进行初步人体解析, 得到粗解析特征; 然后构建边缘轮廓模块, 结合上采样后的全局和 局部特征得到人体边缘轮廓; 再基于着装姿态定义着装姿态损失函数, 通过姿态估计模块提取人体姿态特征; 最后 联合粗解析特征、边缘轮廓和姿态特征, 并定义结构损失和人体解析损失的组合函数输出精确的解析结果. 在多个数 据集上的实验结果表明, 该模型的 mIoU 评测指标提高了 $1.96 \%$, 在人体的着装姿态和部位遮挡等方面获得了更准确 的语义分割结果, 能有效地提高着装人体解析的精度.
\end{abstract}

关键词: 人体解析; 着装场景; 姿态估计; 边缘轮廓; 语义分割

中图法分类号: TP391.41 DOI: 10.3724/SP.J.1089.2021.18683

\section{Accurate Human Parsing Model by Edge Contour and Pose Feature}

\author{
Gan $\operatorname{Lin}^{1)}$, Liu Li ${ }^{1,2) *}$, Liu Lijun ${ }^{1,2)}$, Fu Xiaodong ${ }^{1,2)}$, and Huang Qingsong ${ }^{1,2)}$ \\ 1) (Faculty of Information Engineering and Automation, Kunming University of Science and Technology, Kunming 650500) \\ ${ }^{2)}$ (Computer Technology Application Key Laboratory of Yunnan Province, Kunming 650500)
}

\begin{abstract}
To address the problem of low accuracy and precision of human parsing due to human pose, edge contour, complexity of clothing accessories and occlusions of human pose joints in dressing scenes, an accurate human parsing model with edge contour and pose features is proposed for dressed human. Firstly, the backbone network based on ResNet-101 is used to represent input human body images and extract the coarse parsing features. Secondly, the edge contour module combining the global and local features after upsampling is constructed to obtain the edge contour of human body. Then, the defined human pose loss function based on human pose is added into pose estimation module to acquire pose features. Finally, coarse parsing feature, edge contour and pose features are integrated into accuracy parsing module, and the accurate human parsing results are output by the combined function of structure loss function and human parsing loss function. The experimental results show that the proposed model can effectively improve $1.96 \%$ of mIoU and accuracy on human datasets with more accurate segmentation results for different poses and occlusions of the human body parts.
\end{abstract}

收稿日期: 2020-09-27; 修回日期: 2021-03-12. 基金项目: 国家自然科学基金(61862036, 61962030, 81860318); 云南省中青年学 术和技术带头人后备人才培养计划(201905C160046). 甘霖(1996一), 男, 硕士研究生, 主要研究方向为图形图像处理; 刘骊(1979一), 女, 博士, 教授, 硕士生导师, CCF 高级会员, 论文通讯作者, 主要研究方向为计算机图形学、图像处理; 刘利军(1978一), 男, 硕士, 副教授, CCF 会员, 主要研究方向为图像处理、云计算、信息检索; 付晓东(1975一), 男, 博士, 教授, 博士生导师, CCF 高级会员, 主 要研究方向为服务计算、决策理论与方法; 黄青松(1962一), 男, 硕士, 教授, 硕士生导师, 主要研究方向为机器学习、数据挖掘、智 能信息系统. 
Key words: human parsing; dressing scene; pose estimation; edge contour; semantic segmentation

人体解析 ${ }^{[1]}$ 旨在对人体图像进行逐像素识别, 理解图像中人体各个部分的语义, 广泛应用于行 人重识别、视频监控和人物动作分析. 目前, 人体 解析方法主要有基于特征增强、多任务学习、人体 结构和生成对抗网络 4 种思路 ${ }^{[2]}$. 其中, 基于特征 增强和基于多任务学习 ${ }^{[3]}$ 的方法通过结合多维度 的特征对背景复杂的场景进行人体解析；基于人体 结构的方法 ${ }^{[4]}$ 则是针对运动场景，通过人体结构加 强对人体部位的识别; 基于生成对抗网络的方法 ${ }^{[5]}$ 侧重针对野外和监控视频等场景, 实现跨域解析.

人体解析是一项细粒度的解析任务, 其精确 程度直接影响了其应用性. 基于多任务学习的方 法能够有效地结合多个子任务, 以相互辅助的方 式提高解析的精度. $\mathrm{Li}$ 等 ${ }^{[3]}$ 提出结合人体解析和边 缘检测的多任务网络, 通过结合边缘特征和人体 部位特征提高解析结果的精度, 实现人体的精确 解析; Kikuchi 等 ${ }^{[6]}$ 将姿态估计网络合并到端到端 的人体解析网络中, 以便跨域传递姿态的特征, 实 现了姿态特征辅助人体解析, 以获得精确的解析 结果.

近年来，服装网络购物受到人们的极大欢迎， 呈现出移动化、普及化、全球化的趋势，网络上涌 现出大量不同着装场景的服装图像; 针对着装场 景中的服装图像的研究主要集中于服装分类 ${ }^{[7]}$ 、服 装检索 ${ }^{[8]}$ 、服装推荐以及虚拟试衣等方面. 这些方 法不仅面向大量着装图像, 而且需要聚焦于图像 中以人为中心的区域. 因此, 着装场景中的人体解 析不仅能精确地识别图像中人体各个部位区域的 语义, 而且能有效地为上述工作提供先验知识, 更 好地满足实际应用中的需求.

区别于其他场景，着装场景中的服饰类别繁 多, 复杂的背景和服饰纹理会对人体边缘轮廓提 取产生干扰; 而且着装姿态角度各异, 身体部位存 在重叠、交叉等情况，导致人体部位相互干扰. 此 外，着装人体图像中服饰会对人体部位产生遮挡， 影响人体部位解析的精度. 因此, 着装人体解析仍 存在以下难点：(1) 由于着装人体图像中服装款式 多样，不同服饰的纹理复杂，难以捕获边缘特征， 且图像中复杂的背景会影响边缘轮廓提取的准确 率，导致相邻人体部位的边缘像素存在混淆; (2) 由于着装图像中的人体姿态存在重叠和交叉, 且 关节点之间相互干扰、难以精确定位，使着装人体 关节部位易混淆，导致人体解析精度不高; (3) 着
装人体图像中服饰标签占比大，服饰往往覆盖全 身重要关节，仅考虑姿态信息会由于缺乏服饰边 缘信息导致对人体四肢等部位造成误判; 仅考虑 边缘轮廓特征会由于缺乏人体关节的位置信息而 导致遮挡附近区域出现标签混淆, 使人体解析结 果的精度不高

针对以上问题, 本文首先构建边缘轮廓模块, 以减少背景对边缘提取的干扰，提高部分语义边 界定位的准确率, 为人体解析提供更精确的边缘 关键点信息; 然后添加定义了着装姿态损失函数 的姿态估计模块, 以区分易混淆姿态，有效定位关 节点, 辅助人体部位识别; 最后通过结合边缘轮 廓、姿态特征和粗解析特征进行人体的精确解析, 并引人结构损失函数, 以解决人体的不同姿态和 部位遮挡等问题, 提高着装人体解析的精度.

\section{1 相关工作}

人体边缘信息能够有效地辅助解析任务，帮 助解析模型更好地理解人体语义. Gong 等 ${ }^{[9]}$ 设计 一个集成人体解析和边缘检测的分组网络(part grouping network, PGN), 利用共享语义信息使人 体解析和边缘检测相互受益, 实现人体精确解析; Ruan 等 ${ }^{[10]}$ 提出基于边缘感知的上下文嵌人网络 (context embedding with edge perceiving, CE2P), 不仅有效地结合边缘信息与人体解析, 还减小特 征细节丢失对解析结果的影响, 实现了边缘检测 与人体解析的互补; Huang 等 ${ }^{[11]}$ 采用密集连接的多 孔空间金字塔，结合全局和局部信息实现人体解 析; 虽然上述方法在人体解析和边缘提取上取得 了较好的效果, 但由于着装人体图像中的服饰种 类不一, 款式各样的服装及其纹理会影响轮廓边 界的定位, 而且图像中的背景元素对边缘轮廓识 别存在干扰, 导致难以学习边缘特征. 为了解决上 述问题, 本文以 ResNet-101 $1^{[12]}$ 为主干网络. 通过构 建的边缘轮廓模块, 融合具有全局信息的底层特 征和具有局部信息的高维度特征提高人体轮廓的 准确率, 减少解析结果中由于各个语义边缘难以 准确定位造成的边缘混乱、细节丢失等问题.

姿态特征能有效地定位人体关节点，提高后 续人体解析的精度. Xia 等 ${ }^{[13]}$ 联合姿态估计和人体 解析 2 个子任务, 通过人体解析模块进行姿态校 正，同时利用姿态特征辅助人体解析，获得了精确 
的人体解析结果; Nie 等 ${ }^{[14]}$ 通过反复迭代人体解析 和姿态估计 2 个任务优化特定的模型参数, 以姿态 促进人体解析, 有效地提升了人体解析的精度和 姿态估计的准确率; Liang 等 ${ }^{[15]}$ 提出的联合人体解 析和姿态估计的网络模型(joint human parsing and pose estimation network, JPPNet)能够在预测人体 姿态信息的同时进行人体解析, 采用细化模块对 姿态结果和解析结果进行互补, 实现精确的人体 解析. 尽管这些方法在人体解析任务中取得了一 定成果，但由于着装姿态中人体部位往往会交织 在一起, 难以区分不同姿态, 易混淆人体部位的语 义特征. 为了解决上述问题, 本文针对着装姿态遮 挡和交叉的特点, 在构建的姿态估计模块中, 通过 引人着装姿态损失函数加强对关节点的识别, 进 一步提高人体关节定位的准确率.

人体部位信息对解析至关重要, 合理地利用 人体结构能够加强对人体部位的识别. Gong 等 ${ }^{[4]}$ 提出自监督敏感结构的学习方法, 从全局上下文 的角度整合人体关节的结构信息, 实现了人体解 析; Xia 等 ${ }^{[16]}$ 采用与或图的结构对人体解析各个语 义进行组合, 最后选取置信度最高的结果, 实现人 体精确解析; Wang 等 ${ }^{[17]}$ 结合卷积神经网络和人体 组成部位, 实现基于人体层次结构的人体解析; $\mathrm{Li}$ 等 ${ }^{[18]}$ 通过全局和局部特征减小细节丢失对解析结 果的影响, 实现多人人体解析; Ye 等 ${ }^{[19]}$ 采用由粗到 精的人体解析网络实现了精确人体解析; Liu 等 ${ }^{[5]}$ 采 用生成对抗网络的方法, 通过生成器和鉴别器实 现跨域人体解析; Zhao 等 ${ }^{[20]}$ 提出一个嵌套对抗网 络(nested adversarial network, NAN), 通过 3 个子 网络进行嵌套训练, 获得多人人体精确解析结果; Fang 等 ${ }^{[21]}$ 基于人体结构相似性对已有的解析结果 进行迁移, 实现半监督人体解析; $\mathrm{Wu}$ 等 ${ }^{[22]}$ 通过注 释简单的关键点进行人体解析, 降低数据集标注 的难度, 获得了简单人体解析的结果; Chen 等 ${ }^{[23]}$ 通过约束颜色和类别标签的一致性, 实现弱监督 人体解析. 此外, 文献[1]以 ResNet-101 为主干网 络, 结合姿态估计和注意力机制进行人体解析; Zhang 等 ${ }^{[2]}$ 提出一个相关性解析器 (correlation parsing machine, CorrPM), 利用边缘、姿态和解析 3 个任务的特征进行人体解析, 获得了精确的解析 结果. 受到文献 $[4,24]$ 的启发, 本文提出结合边缘 轮廓和姿态特征进行人体精确解析的思路解决遮 挡问题. 区别于以上方法, 本文利用全局上下文信 息构建边缘轮廓模块, 提高边缘轮廓提取的准确 率; 针对不同着装姿态添加着装姿态损失的姿态
估计模块, 以提高识别率; 为进一步提升解析的精 度, 融合姿态特征提供的关节点信息、边缘轮廓提 供的边缘关键点信息, 以及主干网络提取的粗解 析特征构建精确解析模块, 并引人定义的结构损 失函数对解析特征进行约束.

\section{2 本文模型概述}

图 1 所示为本文模型流程, 其核心是针对着装 场景的特殊性提取精确的姿态和轮廓特征, 并利 用其上下文信息辅助人体精确解析. 步骤如下:

Step1. 构建的边缘轮廓模块解决相近部分的边缘 混淆问题, 提高解析结果中边缘轮廓的准确率;

Step2. 引人着装姿态损失函数, 通过构建的姿态 估计模块提高人体关节点识别的准确率;

Step3. 将提取的人体粗解析特征、边缘轮廓和姿态 特征进行融合, 并通过定义的结构损失和解析损失函 数, 提高语义分割的准确率和人体解析的精度.

\section{3 人体精确解析模型}

着装场景中视觉元素繁多且复杂, 仅靠单一 的主干网络难以得到精确的解析结果. 为了进一 步区分着装场景中人体各部位的语义, 加强解析 结果的精确性, 本文采用多任务学习的方式构建 了包括主干网络、边缘轮廓和姿态估计的人体精确 解析模型.

\section{1 主干网络}

由于多任务网络模型的网络层数过深不能很 好地学习深度特征, 本文采用 ResNet-101 作为主 干网络表征输人着装人体图像, 并引人池化金字 塔提取全局上下文信息. 首先对 Res5 提取的共享 深度特征进行池化, 生成规模为 $1 \times 1,2 \times 2,3 \times 3$, $6 \times 6$ 的全局上下文特征; 然后通过双线性插值对 全局上下文特征进行上采样, 使其与原始特征保 持相同的大小, 并与原始特征进行特征融合; 最后 通过 $1 \times 1$ 卷积减少通道并集成多尺度的上下文信 息, 得到融合特征.

由于多次卷积池化会降低图像分辨率, 而着 装场景中的细节特征往往在局部区域中, 从而丢 失语义之间的细节信息. 为了提高解析的细节效 果, 本文在池化金字塔后添加高分辨率补偿模块, 将中间层提取到的底层视觉特征嵌人高层语义特 征中补充细节, 提高解析的精度. 如图 1 所示, 首 先将 Res 2 提取的共享深度特征通过 $1 \times 1$ 卷积提取 


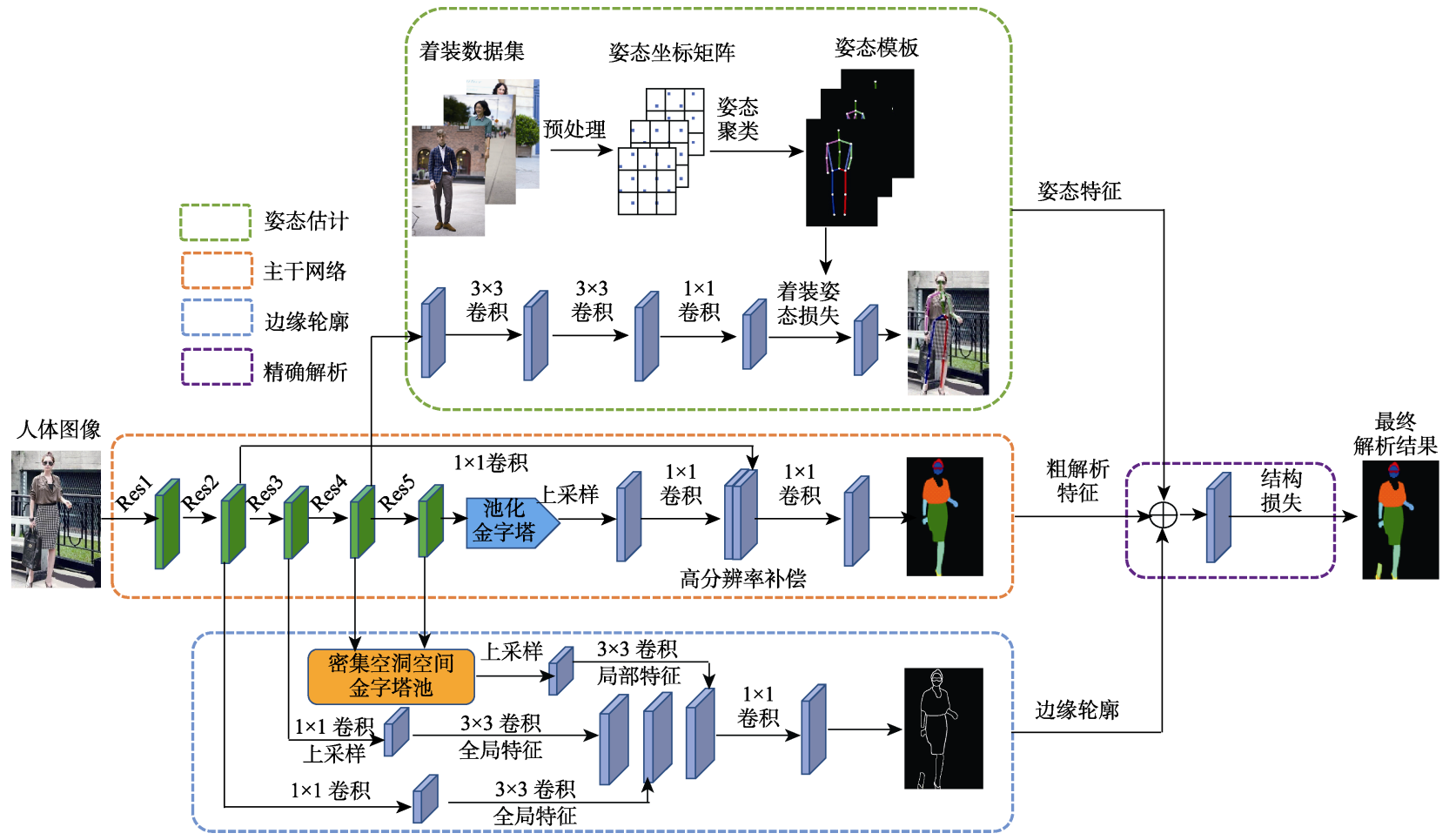

图 1 本文模型流程

具有高分辨率的细节特征; 然后采用双线插值对 融合特征进行上采样，通过 $1 \times 1$ 卷积之后与具有高 分辨率的细节特征进行级联; 再将级联之后的特 征送人 $1 \times 1$ 卷积, 以融合细节特征和全局上下文特 征; 最后通过 $1 \times 1$ 卷积得到粗解析特征.

\section{2 边缘轮廓}

准确的边缘轮廓可为人体解析提供完整的边 缘约束, 提高解析结果的精度. 虽然现有的结合边 缘提取和人体解析的方法取得了较好的效果, 但 是由于着装场景下服饰种类不一, 款式各样的服 装和纹理会影响轮廓边界的定位, 而且背景元素对 边缘轮廓识别产生干扰, 导致难以学习边缘特征. 为了解决上述问题，通过上下文信息处理复杂的着 装人体图像, 以减少服饰纹理和复杂的背景对轮廓 提取的影响; 此外, 还引人密集空洞空间金字塔池 (dense atrous spatial pyramid pooling, DenseASPP)模 块 (由 5 个 $1 \times 1$ 空洞卷积和 5 个 $3 \times 3$ 空洞卷积组成, 卷积的扩张率分别为 $3,6,12,18$ 和 24 . 每个卷积 层的输出与输人的特征图进行级联之后, 再将其 输人下一个扩张层), 以提高网络训练的效率.

边缘轮廓的提取过程包括 4 个阶段：(1) 将 Res4 和 Res5 提取到的共享特征送人密集空洞空间 金字塔池模块，利用该模块来提取特征，对提取到 的特征进行上采样，再通过 $3 \times 3$ 的卷积提取局部 特征; (2) 通过提取具有细粒度信息的全局特征补
充细节信息, 提高轮廓特征的准确率, 将 Res2 和 Res3 提取到的共享深度特征通过 $1 \times 1$ 的卷积提取 细粒度的特征, 并对其进行上采样, 使细粒度特征 大小保持一致; (3) 将细粒度特征送人 $3 \times 3$ 的卷积 提取全局信息，得到全局特征；(4) 将得到的全局 和局部特征进行级联，通过 $1 \times 1$ 卷积融合全局和局 部信息, 最终获得准确的边缘轮廓特征.

边缘轮廓模块中, 边缘损失函数在每个像素 上将预测边缘结果和边缘标注进行计算. 由于边 缘和边缘像素的分布有很大的偏差, 因此采用交 叉熵损失作为边缘损失函数，即

$$
L_{\text {edg }}=-X_{+} \sum_{i \in Y_{-}} \ln \left(1-\hat{y}_{i}\right)-X_{-} \sum_{i \in Y_{+}} \ln \left(\hat{y}_{i}\right)
$$

其中, $i$ 表示第 $i$ 个像素点, $\hat{y}_{i}$ 表示第 $i$ 个像素点的 预测结果; 定义 $Y$ 为真实标注点的集合, $Y=$ $\left(y_{i}, i=1, \cdots,|Y|\right), y_{i} \in(0,1) ; Y_{+}$为正样本像素点集 合， $Y_{+}=\left\{y_{i}, y_{i}>\gamma\right\}, \gamma$ 表示损失计算的阈值; $Y_{-}$为 负样本像素点集合, $Y_{-}=\left\{y_{i}, y_{i}=0\right\} ; X_{+}$为正样本 集合所占比例, $X_{+}=\left|Y_{+}\right| /\left(\left|Y_{+}\right|+\left|Y_{-}\right|\right) ; X_{-}$为负样本 集合所占比例, $X_{-}=\left|Y_{-}\right| /\left(\left|Y_{+}\right|+\left|Y_{-}\right|\right)$.

\section{3 姿态估计}

\subsection{1 姿态聚类}

区别于其他场景下的人体姿态，着装人体姿 态容易出现人体部位交织在一起的情况, 造成人 
体各个关节点之间相互遮挡. 为此, 通过定义姿态 模板加强对易混淆人体部位的识别, 减少对人体 关节点的误识别, 提高姿态估计准确率.

着装人体图像中关键点的选取能有效地辅助 识别人体关节点, 进一步提高人体关节点定位的 准确率. 此外, 着装场景中的姿态变化主要取决于 细节区域, 如头、肩等关节的遮挡、变化较小, 因 此本文将易遮挡、混淆的 8 个人体关节点(左/右肘、 左/右腕、左/右膝和左/右踝)作为关键点, 并选取包 含关键点的着装图像进行姿态聚类.

针对姿态坐标归一化直接影响姿态聚类结果 的问题, 本文基于文献[25], 在姿态处理之前通过 预处理对聚类图像进行剪裁. 首先, 通过 Mask $\mathrm{R}-\mathrm{CNN}^{[26]}$ 预测着装人体图像中的人物边界框; 然 后, 利用边界框对着装图像中的人物进行感兴趣 区域(region of interest, RoI)定位, 选择人物主要出 现的 RoI, 并通过已经标注好的关节点坐标判断其 是否在 RoI 内, 如不在, 则需要重新选取 RoI 使裁 剪的结果为完整的人体图像; 再根据姿态坐标与 RoI 之间的距离调整人物的姿态坐标, 通过平移等 操作将其置于 RoI 的中心, 使完整的人体图像居 中; 最后, 设置 RoI 的大小为 $64 \times 64$, 以提高姿态 模板的准确性和归一化姿态坐标.

本文将人体姿态定义为一个矢量集合，定义 人体姿态集合 $\boldsymbol{P}=\left(\boldsymbol{J}_{1}, \boldsymbol{J}_{2}, \cdots, \boldsymbol{J}_{m}\right) \in \mathbb{R}^{M \times 3}$. 其中, $\boldsymbol{J}_{m}=(x, y, v) \in \mathbb{R}^{3}$ 为一个三维向量, 包含人体的一 个关节点的坐标和可见度; $x, y$ 代表一个关节点 的坐标位置， $v$ 表示该关节点的可见度； $M$ 表示 关节点的总个数, 在本文数据集中共定义了 16 个 关节点.

利用着装图像构建姿态集合 $\left(\boldsymbol{P}_{1}, \boldsymbol{P}_{2}, \cdots, \boldsymbol{P}_{n}\right)$, 其中, $n$ 表示训练集的数量. 然后采用 $K$ 均值聚类 的方法, 通过 $\underset{Z}{\arg \min } \sum_{k=1}^{K} \sum_{\boldsymbol{P} \in \boldsymbol{Z}_{k}} D\left(\boldsymbol{P}, \boldsymbol{P}_{\text {mean }} k\right)$ 将姿态列 表集合 $\left(\boldsymbol{P}_{1}, \boldsymbol{P}_{2}, \cdots, \boldsymbol{P}_{n}\right)$ 聚类为 $k(k \leqslant n)$ 个类. 其中, $Z$ 表示所有聚类结果的集合 $Z=\left(Z_{1}, Z_{2}, \cdots, Z_{k}\right)$; $\boldsymbol{P}_{\text {mean } \_k}$ 表示 $\boldsymbol{Z}_{k}$ 中所有姿态平均值的集合; 姿态之 间的差异值定义为

$$
D\left(\boldsymbol{P}, \boldsymbol{P}_{\text {mean } \_k}\right)=\sum_{m=1}^{M}\left\|\boldsymbol{J}_{m}-\boldsymbol{J}_{\text {mean } \_k m}\right\|^{2}
$$

$\boldsymbol{J}_{\text {mean } \_k m}$ 表示 $\boldsymbol{P}_{\text {mean } \_k}$ 中第 $m$ 个关节点的值, $M$ 表 示着装人体图像中所有的关节点, \|\|$^{2}$ 表示欧几里 得度量.
为更好地区分姿态聚类结果中易混淆的着装 姿态, 本文定义不在图像中的关节点的可见度为 0 , 被遮挡关节点的可见度为 1 , 未被遮挡的关节 点的可见度为 2 . 这不仅可以区分被遮挡关节点和 不在图像中关节点, 加强网络模型对遮挡、易混淆 关节点的识别, 而且能够区分被遮挡和未被遮挡 关节点, 减小特殊姿态对姿态聚类的干扰. 针对无 效关节点降低姿态模板准确性问题, 对于不在图 像中的关节点, 将其 $x$ 和 $y$ 坐标设为 0.5 , 能较好 地避免在姿态聚类时关节点坐标对其他关节点坐 标造成的干扰, 关节点可见度表示为

$$
\boldsymbol{J}_{m}= \begin{cases}(x, y, 2), & \text { 未被遮挡 } \\ (x, y, 1), & \text { 被遮挡 } \\ (0.5,0.5,0), & \text { 不在图像中 }\end{cases}
$$

其中, $x$ 和 $y$ 表示关节点归一化之后的像素位置.

$K$ 均值聚类之后，使用每个集合中姿态的平均 值 $\boldsymbol{P}_{\text {mean } \_k}$ 构成姿态模板. 为了减少可见度低的关 节点对姿态聚类的影响, 提高姿态模板的准确性, 将 $\boldsymbol{P}_{\text {mean } \_k}$ 集合中 $v>0.5$ 的关节点设置为有效关节 点. 最终的姿态模板为 $\boldsymbol{P}_{\text {mean } \_k}$ 中有效关节点的集 合. 由于关节点可见度的取值和 $k$ 的初始值对 $K$ 均 值聚类的结果影响较大, 通过多次实验设置及结 果的对比, 选择式(3)中可见度的取值和定义 $k=4$ 较为合理.

\subsection{2 姿态估计方法}

姿态信息能够有效地进行人体定位, 辅助着 装人体解析任务. 目前, 通用的姿态估计方法主要 基于堆叠沙漏网络 ${ }^{[27]}$, 虽然能够取得较好的姿态估 计效果, 但由于网络结构复杂, 其直接与人体解析 进行多任务学习时不利于网络模型的收玫. 为了更 好地嵌套到联合解析网络中, 本文将 ResNet-101 作 为共享的主干网络提取共享特征, 加人姿态估计 模块和着装姿态损失函数, 以提取姿态特征. 将 Res4 提取的共享特征输人姿态估计模块, 通过 6 个卷积大小为 $3 \times 3$ 、卷积核数为 256 的卷积层提取 姿态特征; 通过卷积大小为 $1 \times 1$ 、卷积核数为 16 的卷积层得到人体姿态特征; 再针对着装姿态的 自遮挡和交叉问题, 定义姿态结构损失函数和着 装姿态损失函数, 以提高人体关节点识别的准确 率, 得到最终的姿态特征.

姿态结构损失函数通过计算姿态得分图与姿 态标注之间的欧几里得损失得到, 定义为

$$
L_{\mathrm{e}}=\frac{1}{2 M} \sum_{m=1}^{M}\left\|C_{m}^{\mathrm{p}}-C_{m}^{\mathrm{gt}}\right\|_{2}^{2}
$$


其中, $M$ 为关节点的总数, $C_{m}^{\mathrm{p}}$ 表示第 $m$ 个关节在 图像中的像素坐标. $C_{m}^{\mathrm{gt}}$ 表示第 $m$ 个关节在图像中 真实的像素坐标.

不同的姿态模板对应的姿态特征存在差异, 通过提取不同的姿态特征可获得更高的姿态估计 准确率. 因此, 本文采用 Softmax 损失将着装姿态 损失函数定义为

$$
L_{\text {pose_cluster }}=-\sum_{k=1}^{4} Z_{k} \ln P_{k}
$$

其中, $Z_{k}$ 为一个 $1 \times 4$ 的向量, 该向量的大小由姿 态模板的类别数所定; $P_{k}$ 表示该图像中的人体姿 态属于第 $k$ 种姿态模板的概率.

\section{4 精确解析}

为减少遮挡对人体解析精度的影响, 获取更 加精确的人体解析结果, 本文利用姿态特征、边缘 轮廓特征和粗解析特征进行联合解析, 并定义结 构损失和人体解析损失的组合损失函数来提升解 析结果的精度.

图 2 所示为本文构建的精确解析模块. 首先将 粗解析特征 $F_{\mathrm{p}}$ 、边缘轮廓特征 $F_{\mathrm{e}}$ 和姿态特征 $F_{\mathrm{k}}$ 进 行级联, 并通过卷积将级联后特征映射为 $N \times C$ 大 小, 其中, $N$ 为图像高乘以宽, $C$ 为特征的维度; 然后将粗解析特征通过卷积映射为 $C \times N$ 大小的 特征; 最后将 2 个特征矩阵进行相乘, 并通过 Softmax 归一化得到联合特征.

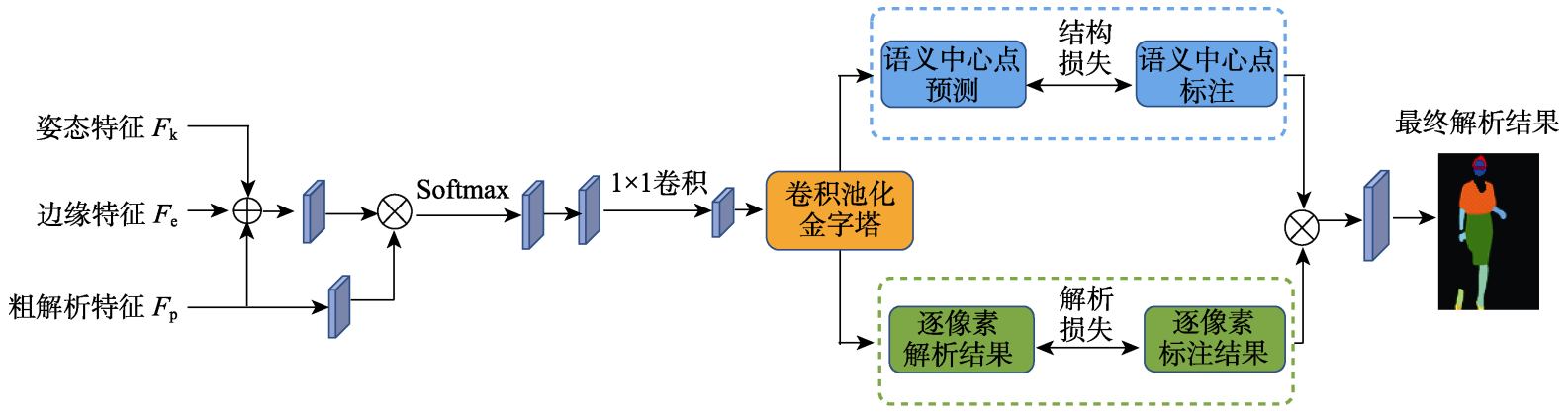

图 2 精确解析模块

图 2 中构建了 4 个卷积大小不一的卷积层, 通 过卷积核大小为 $3 \times 3,5 \times 5,7 \times 7$ 和 $9 \times 9$ 的卷积来分 别获取局部上下文信息，并增加接收信息区域的 大小; 然后将提取到的特征送人一个卷积大小为 $1 \times 1$ 、卷积核数为 256 的卷积层中生成细化特征; 再使用空洞空间卷积金字塔池(atrous spatial pyramid pooling, ASPP)生成精确的人体解析特征; 最 后引人精确解析损失函数, 该函数是结构损失和 解析损失的组合, 定义为

$$
L_{\text {finer_parsing }}=L_{\text {parsing }} L_{\text {structure }}
$$

其中， $L_{\text {structure }}$ 表示结构损失, 用于计算各个语义中 心区域与标注结果中语义中心区域之间的距离. 即

$$
L_{\text {structure }}=\frac{1}{2 N} \sum_{i=1}^{N}\left\|S_{i}^{\mathrm{p}}-S_{i}^{\mathrm{gt}}\right\|_{2}^{2}
$$

其中， $S_{i}^{\mathrm{p}}$ 表示预测图像中第 $\mathrm{i}$ 个语义标签的中心 点位置; $S_{i}^{\mathrm{gt}}$ 表示标注图像中第 $i$ 个语义标签的中 心点位置; $N$ 表示语义标签的数量. 对于标注图 像中没有的语义标签, 将 $L_{\text {structure }}$ 值重置为 0 . 以裙 子和连衣裙为例, 在解析结果中会出现将连衣裙 解析成了上衣和裙子 2 部分的情况, 主要是因为
上、下部分存在纹理差异或有腰带等配饰遮挡的情 况. 结构损失将不存在的语义标签中心(上衣, 裙 子)置为 0 , 强化网络模型对语义标签的识别.

$L_{\text {parsing }}$ 表示解析结果与解析注释之间的交叉 熵损失，即

$$
L_{\text {parsing }}=-\frac{1}{b} \sum_{i=1}^{b} \sum_{n=1}^{N} 1\left\{y_{i}=n\right\} \ln \left(p_{n}\right)
$$

其中, $b$ 表示图像中像素点总个数; $N$ 表示语义 标签类别数; $y_{i}$ 表示第 $i$ 个像素点的类别; $1\left\{y_{i}=n\right\}$ 表示如果第 $i$ 个像素点的类别为 $n$, 其值 取 1 , 否则其值取 $0 ; \ln \left(p_{n}\right)$ 表示预测为第 $n$ 类语 义标签的概率.

对网络模型不断进行迭代训练优化网络模型. 通过式(7)计算结构损失; 通过式(8)计算人体解析 损失; 通过式(6)计算组合损失; 最后不断地对网 络模型进行迭代直到收玫, 得到最终的人体解析 结果.

本文构建的网络模型具有多个模块, 整体损 失函数 $L$ 为多个损失函数加权系数之和, 定义为

$$
L=L_{\text {rough_parsing }}+\alpha L_{\text {key }}+\beta L_{\text {edge }}+L_{\text {fine_parsing }}
$$




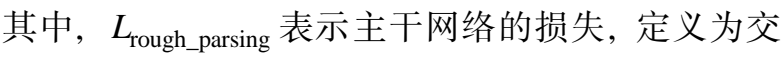
叉熵损失; $L_{\text {edge }}$ 表示边缘轮廓模块的损失; $L_{\mathrm{key}}$

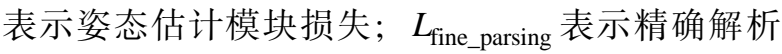
模块的损失. 为了防止解析任务损失完全占据了 主导地位而影响了边缘轮廓和姿态估计任务的学 习, 本文给这 2 个子任务设置更高的权重, 最终设 置 $\alpha=70, \beta=2$.

人体精确解析模型算法步骤如下.

Step1. 主干网络. 以 ResNet-101 为主干网络, 利 用池化金字塔提取多尺度特征，并通过特征补偿来提升 解析特征的细节信息，得到粗解析特征.

Step2. 边缘轮廓. 结合全局和局部信息进行特征 提取, 通过式(1)计算边缘轮廓的损失, 加强语义边界的 定位准确率, 得到边缘轮廓特征.

Step3. 姿态估计:

Step3.1. 通过式(3)对人体关节点进行数值化表 示, 方便进行聚类计算, 并采用 $K$ 均值聚类的方法, 通 过式(2)对人体姿态进行聚类，得到着装姿态分类;

Step3.2. 针对着装姿态的特殊性, 用式(5)来加 强对人体关节点的识别, 并通过姿态估计模块提取精确 的姿态特征.

Step4. 精确解析：

Step4.1. 通过精确解析模块, 利用粗解析特征、 姿态特征和边缘轮廓特征辅助人体部位的识别提升解 析性能, 减少遮挡对人体解析的影响;

Step4.2. 通过式(7)(8)定义结构损失函数和人体 解析损失函数, 进一步加强网络模型对易混淆语义标签 的识别能力. 在多次的迭代训练后, 得到最终的人体解 析结果.

\section{4 实验结果与分析}

\section{1 实验数据集}

本文实验选用 Intel Core i9-9900k CPU@ 3.60 GHz, GPU 2080Ti, 32 GB DDR4 2666 MHz RAM 的硬件平台，采用 PyCharm 集成式开发环境.

本文以服装联合解析 (clothing co-parsing, CCP)数据集 ${ }^{[28]}$ 中的时尚着装图像为基础, 构建了 一个包含 19 个语义标签的着装人体图像数据集. 为了更好地体现着装特点, 还选取 DeepFashion ${ }^{[7]}$ 和网络上搜索的图像对着装人体图像数据集进行 补充, 共收集了 21210 幅图像. 其中, 男性图像 9710 幅, 女性图像 11500 幅.

本文使用 LabelMe 工具 ${ }^{[29]}$ 对人体着装图像进 行精细的语义标注, 该数据集中包含 19 个语义标 签: 帽子、头发、太阳镜、上衣、连衣裙、外套、
袜子、裤子、手套、围巾、短裙、连衫裤、面部、 右臂、左臂、右腿、左腿、右鞋和左鞋. 数据集排 名前 10 种不同类别的语义标签的数量分布如图 3 所示, 可以看出, 除了人体部位标签(如头发、左/ 右手、左/右鞋等)之外，帽子等常见配饰出现较多.

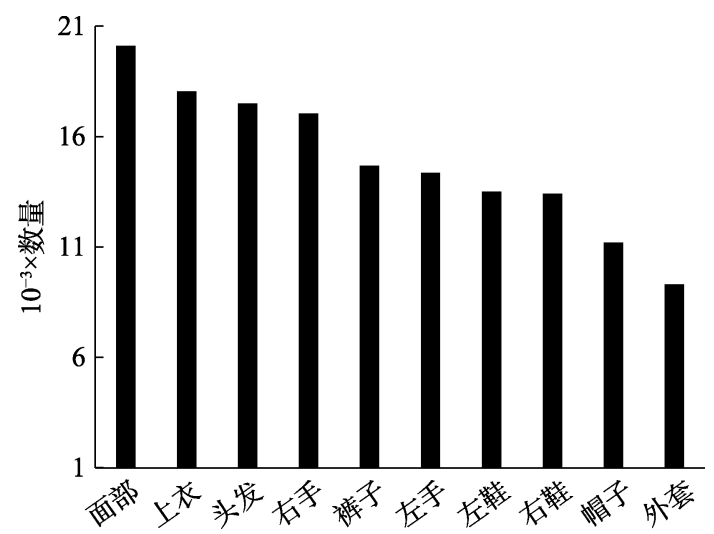

图 3 数据集中标签数量分布

此外, 为了更好地适应人体特征, 还标注了 16 个关节点的位置和可见性, 并区分左右. 在测 试时, 既要正确估计关节点, 又要正确匹配左右. 数据集中原始图像的 $80 \%$ 用于训练, $5 \%$ 用于验证, 15\%用于测试, 所有图像统一调为 JPG 格式.

针对聚类图像的优劣直接影响姿态聚类结果 的问题, 本文利用数据集中图像进行姿态聚类(第 3.3.1 节), 通过预处理步骤对数据集中包含了关键 点的着装图像进行剪裁, 并将 RoI 的大小调整为 $64 \times 64$ 像素.

\section{2 边缘轮廓与姿态估计性能分析}

\subsection{1 边缘轮廓实验结果}

着装图像的人体轮廓预测结果如表 1 所示. 可 以看出, $\mathrm{CE} 2 \mathrm{P}^{[10]}$ 预测结果中存在以下 2 个问题: (1) 边缘细节丢失, 如图像 1 中上衣和外套的色块较为 相近导致其边缘结果中边缘丢失, 图像 2 中的边缘 结果存在裙子的下摆边缘属性丢失; (2) 边缘信息 识别错误, 如图像 2 边缘结果中腿部的边缘信息产 生了大量的误识别, 图像 3 边缘结果中肩部附近的 边缘信息出现了混乱, 图像 4 边缘结果中裙子上出 现误识别的边缘. 表 1 的实验对比结果表明, 本文 模型的人体轮廓结果较好.

\subsection{2 姿态聚类有效性分析}

本文通过 $K$ 均值聚类的方法利用姿态标注文 件进行聚类. 由于聚类姿态模板是针对着装姿态 易混淆的问题, 如果姿态模板选取过多就失去了 聚类的意义, 所以本文设置 $K$ 的取值范围为 1 6. 
表 1 人体轮廓结果及对比实例

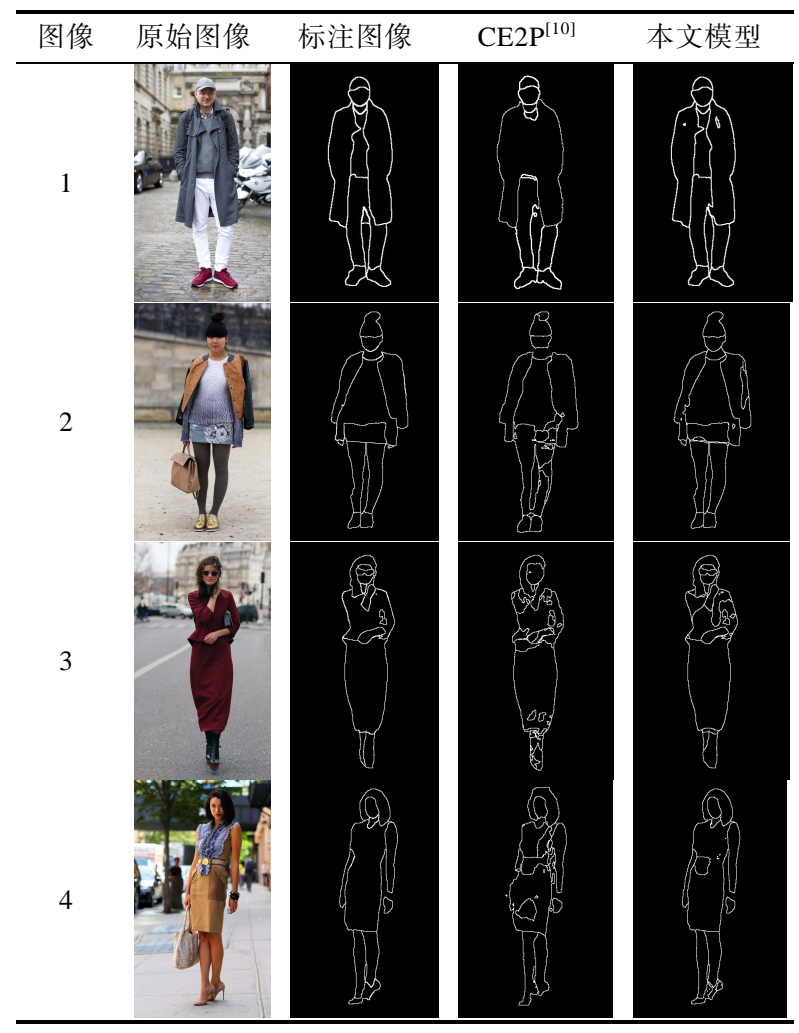

由于关节点可见度 $v$ 的设置与 $K$ 的取值关联性强, 且对准确率影响较大, 为此, 通过多组对比实验验 证选取 $K$ 和关节点可见度 $v$ 的最佳设置.

为进一步验证本文处理关节点可见度的有效 性, 以三元组的形式表示关节点可见度 $v\left(t_{1}, t_{2}, t_{3}\right)$. 其中， $t_{1}$ 表示不在图像中关节点的可见度， $t_{2}$ 表示 被遮挡关节点可见度， $t_{3}$ 表示未被遮挡关节点可 见度. 图 4 所示为不同类别数的姿态模板对姿态估 计准确率的影响. 可以看出, 通过多组实验对比, 当姿态类别数变化时, 采用 $v(0,1,2)$ 均取得了最高 的准确率. 因此, 为有效地处理姿态遮挡问题, 本 文将关节点可见度设置为 $v(0,1,2)$. 当姿态模板数 小于或等于 4 时姿态估计的准确率不断上升, 但在 大于 4 之后姿态估计的准确率开始下降，原因是姿 态聚类数量过多，导致难以学习到适当的姿态信 息. 因此, 将姿态类别数设置为 4 较为合理, 能提取 精确的姿态特征, 获得最佳的姿态估计效果.

为进一步说明姿态模板的必要性. 本文对比 了有姿态模板和无姿态模板的姿态估计准确率, 如表 2 所示. 可以看出, 有姿态模板比没有姿态模 板的姿态估计准确率提高了 $2.80 \%$, 说明姿态模板 能够有效地提升姿态估计的准确率.

\section{2 .3 姿态估计实验结果}

本文采用 $\mathrm{JPPNet}^{[15]}$ 中对姿态估计的评估标准

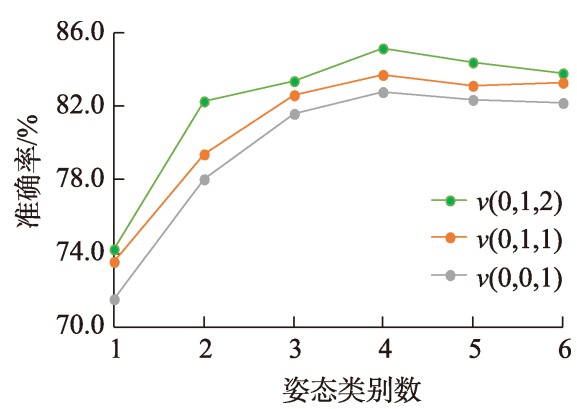

图 4 不同数量姿态类别对姿态预测的影响

表 2 有/无姿态类别对姿态估计准确率的影响 \%

\begin{tabular}{|c|c|c|c|c|c|}
\hline \multirow{2}{*}{ 模板 } & \multicolumn{4}{|c|}{ 姿态类别 } & \multirow{2}{*}{ 总体 } \\
\hline & 头 & 肩 & 腕 & 膌 & \\
\hline 无姿态 & 94.3 & 89.1 & 82.1 & 76.5 & 82.4 \\
\hline 有姿态 & 94.9 & 91.6 & 84.3 & 79.1 & 85.2 \\
\hline
\end{tabular}

进行评估. 表 3 所示为本文与 4 种姿态估计方法的 准确率比较, 可以看出, 与 4 种姿态估计方法中表 现最好的 JPPNet 相比, 本文模型对头部关节的识 别提高 $1.7 \%$, 肩部关节准确率提高 $2.4 \%$, 腕部关 节准确率提高 2.1\%, 总体准确率提高 $1.9 \%$; 与其 他方法相比，本文在识别人体各个关节的准确率 上均有提高.

表 35 种方法准确率比较 $\%$

\begin{tabular}{|c|c|c|c|c|c|}
\hline \multirow{2}{*}{ 方法 } & \multicolumn{4}{|c|}{ 姿态类别 } & \multirow{2}{*}{ 总体 } \\
\hline & 头 & 肩 & 腕 & 膝 & \\
\hline ResNet-101 ${ }^{[12]}$ & 91.2 & 84.3 & 74.9 & 69.5 & 76.5 \\
\hline $\mathrm{CPM}^{[30]}$ & 90.5 & 84.8 & 75.0 & 69.6 & 76.8 \\
\hline Hourglass $^{[27]}$ & 91.2 & 85.7 & 75.5 & 70.5 & 77.5 \\
\hline $\mathrm{JPPNet}^{[15]}$ & 93.2 & 89.2 & 82.2 & 78.1 & 83.3 \\
\hline 本文 & 94.9 & 91.6 & 84.3 & 79.1 & 85.2 \\
\hline
\end{tabular}

着装图像的姿态估计结果如表 4 所示. 可以看 出, JPPNet ${ }^{[15]}$ 的姿态估计结果中存在相似关节混 淆、关节定位有误等问题. 例如, 图像 1 姿态估计 结果将右鞋识别为了左鞋, 而对左鞋没有识别; 图 像 2 姿态估计结果中出现左右鞋混淆; 图像 3 姿态 估计结果中右手的肘关节定位错误; 图像 4 姿态估 计结果中存在膝关节和踝关节定位错误. 由表 4 的 实验结果表明, 本文模型的姿态估计结果较好.

\section{3 人体解析结果与性能分析}

\subsection{1 有效性分析}

本文通过对每个模块进行消融实验证明每个 模块的有效性. 使用像素准确率、平均精度、平均 交并比(mean intersection over union, mIoU)衡量人 体解析的性能. 表 5 中, Rev 表示对训练集中的图 
表 4 姿态估计结果及对比实例

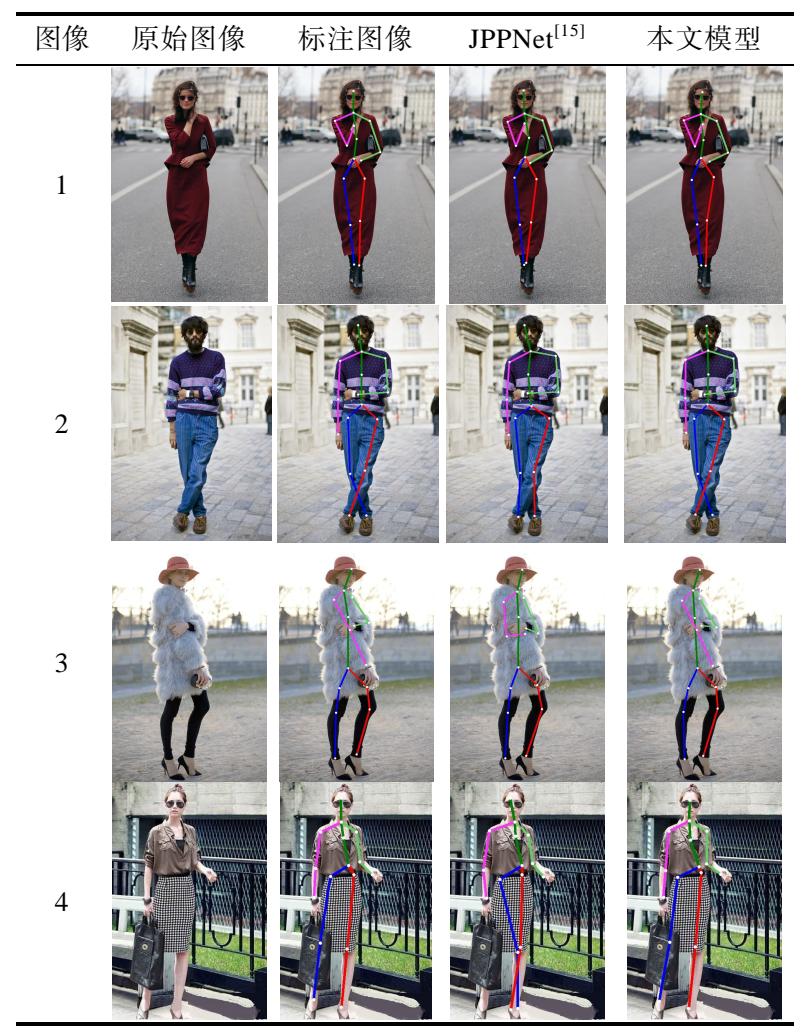

表 5 人体精确解析模型消融实验比较

\begin{tabular}{lccc}
\hline \multicolumn{1}{c}{ 方法 } & 像素准确率 & 平均精度 & mIoU \\
\hline Base & 78.34 & 58.36 & 48.92 \\
Base+P & 81.68 & 61.87 & 51.73 \\
Base+P+E & 84.53 & 64.73 & 53.81 \\
Base+P+E+H & 87.53 & 67.88 & 57.06 \\
Full+Rev & 88.72 & 68.73 & 57.27 \\
\hline
\end{tabular}

像进行了左右翻转; $\mathrm{P}, \mathrm{H}$ 和 $\mathrm{E}$ 分别是姿态估计、精 确解析模块和边缘轮廓模块; Full 表示在主干网络 加人姿态估计、精确解析模块和边缘轮廓模块; Base 表示没有任何模块的主干网络模型, 解析结 果是直接在 ResNet-101 提取的最终特征图进行上 采样所得到. 表 5 结果表明, 每个模块对人体解析 的性能均有提升.

\subsection{2 人体解析实验结果}

为进一步验证本文模型的有效性，在获取公 开源码的基础上, 对本文的数据集重新训练学习, 与 JPPNet ${ }^{[15]}, \mathrm{PGN}^{[9]}$, 文献[3]和 CorrMP ${ }^{[24]}$ 相关方 法进行定性和定量对比. 本文模型的着装人体解 析结果与以上相关方法的对比分析如图 5 所示. 利 用相关方法进行着装人体解析会出现如下问题: （1）当服饰纹理复杂时, 容易使解析结果出现过度 分割和边缘信息丢失, 如图像 5 中服饰与背景的色 块相似时, PGN ${ }^{[9]}$ 和文献[3]的解析结果丢失了边缘
信息；(2) 着装场景下部分姿态会影响人体关节点 识别, 导致错误解析结果, 如图像 6 存在人体双腿 交叉，现有方法对左右鞋的解析产生混淆; (3) 当 人体部位被遮挡时, 难以区分遮挡部位附近区域 的语义, 如图像 3 中腿部被包遮挡时, JPPNet ${ }^{[15]}$ 和 CorrMP $^{[24]}$ 的解析结果中左腿遮挡附近部分出现语 义混淆.

本文通过边缘轮廓模块、姿态估计模块、精确 解析模块解决解析结果不够精确的问题. 利用边 缘轮廓模块结合全局和局部信息提取边缘信息; 通过加人着装姿态损失的姿态估计模板加强对关 节点的识别; 利用定义的结构损失和解析损失函 数的精确解析模块减小遮挡对解析结果精确性的 影响; 使用像素准确率、平均精度和 mIoU 衡量人 体解析的性能.

利用相关方法的开源代码, 在本文数据集上重 新进行训练, 并将其结果与本文模型进行量化对比, 结果如表 6 所示. 可以看出, 本文模型的 mIoU 较其 他方法提升 1.96\%以上, 具有较好的解析结果.

表 6 人体解析相关方法比较

\begin{tabular}{lccc}
\hline \multicolumn{1}{c}{ 方法 } & 像素准确率 & 平均精度 & mIoU \\
\hline JPPNet $^{[15]}$ & 85.93 & 61.45 & 50.63 \\
PGN $^{[9]}$ & 86.51 & 63.73 & 53.53 \\
CE2P $^{[10]}$ & 86.37 & 62.54 & 52.18 \\
CorrPM $^{[24]}$ & 87.35 & 67.13 & 55.31 \\
本文 & 88.72 & 68.73 & 57.27 \\
\hline
\end{tabular}

图 6 所示为本文模型与基于本文数据集重新 训练的 $\mathrm{CE} 2 \mathrm{P}^{[10]}$ 之间的部分语义 $\mathrm{mIoU}$ 对比. 可以 看出，本文模型不仅对上衣、外套等大标签解析性 能有显著提升，而且对眼镜、围巾等小标签的解析 性能也有一定提升.

本文模型时间复杂度为

$$
O\left(\sum_{i=1}^{I} M_{i}^{2} \cdot K_{i}^{2} \cdot C_{i-1} \cdot C_{i}\right) \cdot
$$

其中, $I$ 为网络模型的卷积层数; $i$ 为第 $i$ 个卷积层; $M_{i}^{2}$ 为第 $i$ 个卷积核输出的特征图大小; $K_{i}^{2}$ 为第 $i$ 个卷积核大小; $C_{i}$ 为第 $i$ 个卷积层的输出通道数. 表 7 所示为本文模型和其他相关人体解析方法的 时间复杂度和解析精度对比. 其中, $\mathrm{EEN}^{[11]}$ 是以 ResNet-101 为基础构建的网络, 网络深度 36 层; CE2P ${ }^{[10]}$ 的主干网络是 ResNet-101, 网络深度 22 层; JPPNet ${ }^{[15]}$ 以 ResNet-101 为主干网络, 网络深度 38 层; $\mathrm{NAN}^{[20]}$ 使用 FCN 为主干网络, 网络深度 48 层. 
图像 1
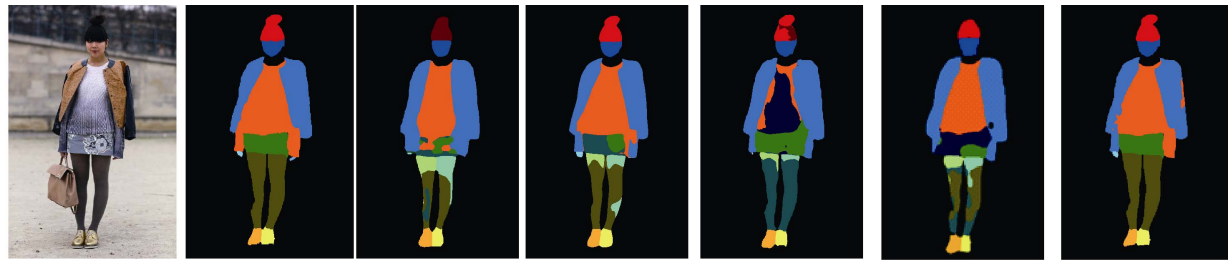

图像 2
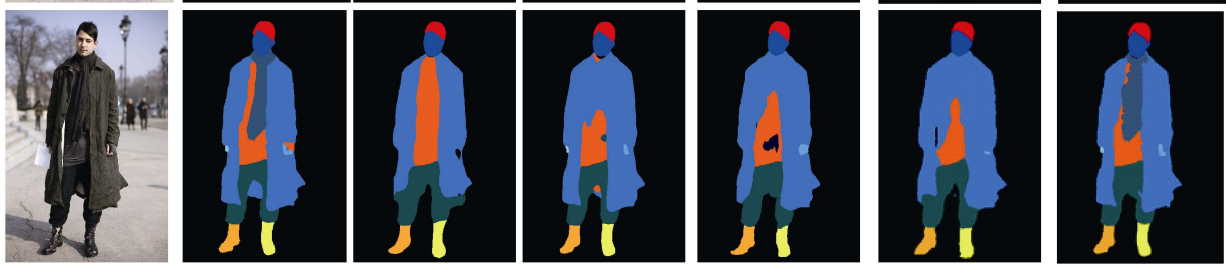

口背景
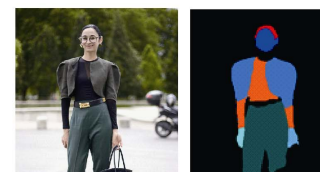

图像 3
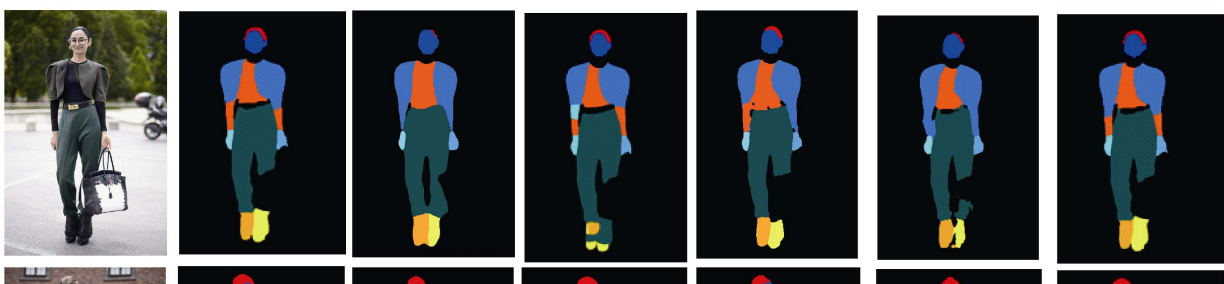

口帽子

图像 4
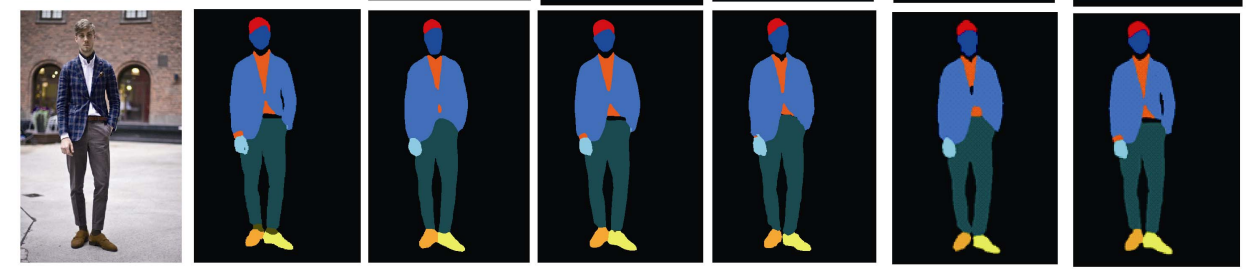

头发
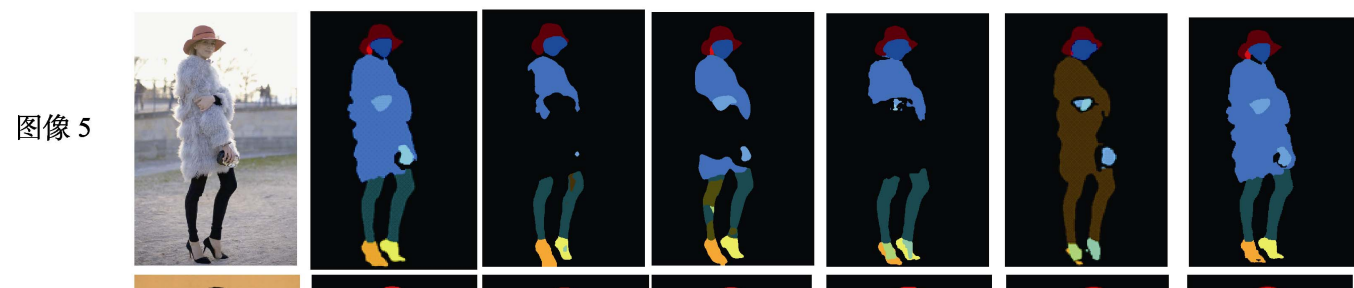

手套

太阳眼镜

$\square$ 上衣

回衣裙

图像 6
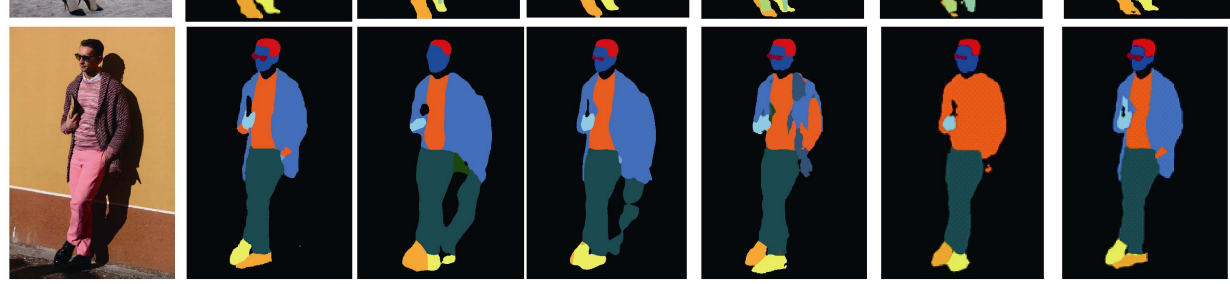

$\square$ 外套

a. 原始图像

b. 标注图像

c. JPPNet ${ }^{[15]}$

d. $\mathrm{PGN}^{[9]}$

e. 文献[3]

f. CorrMP ${ }^{[24]}$

g. 本文

图 5 人体解析结果及对比实例

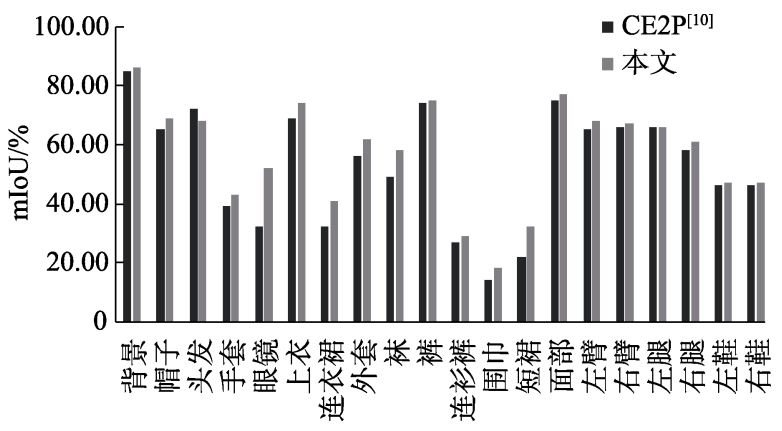

图 6 本文模型与 $\mathrm{CE} 2 \mathrm{P}^{[10]}$ 部分语义 $\mathrm{mIoU}$ 对比

可以看出, 本文模型在拥有较高的解析准确率的 同时，也具有较低的时间复杂度.

\begin{tabular}{lccc}
\multicolumn{2}{c}{ 表 7} & 人体解析方法的时间复杂度和解析精度比较 \\
\hline \multicolumn{1}{c}{ 方法 } & 卷积层数 & 时间复杂度 & $\mathrm{mIoU} / \%$ \\
\hline $\mathrm{EEN}^{[11]}$ & 36 & 中 & 55.53 \\
$\mathrm{CE}^{[10 \mathrm{P}}{ }^{[10]}$ & 22 & 低 & 52.18 \\
$\mathrm{JPPNet}^{[15]}$ & 38 & 中 & 50.63 \\
$\mathrm{NAN}^{[20]}$ & 48 & 高 & 56.09 \\
本文 & 39 & 中 & 57.27 \\
\hline
\end{tabular}

\section{5 结 语}

针对现有方法在着装场景中存在边缘细节丢 失、标签混淆和人体关节点识别不够精确等问题, 本文提出一种结合边缘轮廓和姿态特征的人体精 
确解析模型. 通过边缘轮廓模块解决相邻部分的 边缘混淆问题, 提高了解析结果中边缘轮廓的准 确率; 针对着装姿态的自遮挡、易混淆的问题, 加 人着装姿态损失函数，通过构建姿态估计模块提 高人体关节点识别的准确率; 最后将提取的人体 粗解析特征、边缘轮廓和姿态特征进行融合, 并通 过定义的结构损失函数进一步加强对各个语义部 分的识别. 实验结果表明, 本文模型不仅减少遮挡 对人体解析的影响, 而且加强了网络模型对语义 标签的识别度, 实现了人体精确解析.

本文模型能够较准确地对着装人体图像进行 解析, 但存在部分人体解析结果效果不理想的问 题，尤其是对于纹理丰富的服饰，在背景复杂的情 况下解析准确率不高. 此外, 虽然本文模型取得了 较好的准确率，但时间复杂度仍然较高. 后续将继 续优化网络结构, 重点解决这些问题.

\section{参考文献(References):}

[1] Gao Mingda, Sun Yubao, Liu Qingshan, et al. Posture prior driven double-branch network model for accurate human parsing[J]. Journal of Software, 2020, 31(7): 1959-1968(in Chinese)

(高明达, 孙玉宝, 刘青山, 等. 联合姿态先验的人体精确解 析双分支网络模型[J]. 软件学报, 2020, 31(7): 1959-1968)

[2] Shao Jie, Huang Xi, Cao Kuntao. A review on deep learning techniques applied to human parsing[J]. Journal of University of Electronic Science and Technology of China, 2019, 48(5): 644-654(in Chinese)

(邵杰, 黄茜, 曹坤涛. 基于深度学习的人体解析研究综述 [J]. 电子科技大学学报, 2019, 48(5): 644-654)

[3] Li P K, Xu Y Q, Wei Y C, et al. Self-correction for human parsing[OL]. [2020-09-27]. https://arxiv.org/pdf/1910.09777v1. pdf

[4] Gong K, Liang X D, Zhang D Y, et al. Look into person: self-supervised structure-sensitive learning and a new benchmark for human parsing[C] //Proceedings of the IEEE Conference on Computer Vision and Pattern Recognition. Los Alamitos: IEEE Computer Society Press, 2017: 932-940

[5] Liu S, Sun Y, Zhu D F, et al. Cross-domain human parsing via adversarial feature and label adaptation[C] //Proceedings of the 32nd AAAI Conference on Artificial Intelligence. Palo Alto: AAAI Press, 2018

[6] Kikuchi T, Endo Y, Kanamori Y, et al. Transferring pose and augmenting background for deep human image parsing and its applications[J]. Computational Visual Media, 2018, 4(1): 43-54

[7] Liu Z W, Luo P, Qiu S, et al. DeepFashion: powering robust clothes recognition and retrieval with rich annotations[C] //Proceedings of the IEEE Conference on Computer Vision and Pattern Recognition. Los Alamitos: IEEE Computer Society Press, 2016: 1096-1104

[8] Huang Dongyan, Liu Li, Fu Xiaodong, et al. Clothing retrieval via co-segmentation and feature matching[J]. Journal of Computer-Aided Design \& Computer Graphics, 2017, 29(6): 1075-1084(in Chinese)

(黄冬艳, 刘骊, 付晓东, 等. 联合分割和特征匹配的服装图 像检索[J].计算机辅助设计与图形学学报, 2017，29(6): 1075-1084)

[9] Gong K, Liang X D, Li Y C, et al. Instance-level human parsing via part grouping network[C] //Proceedings of European Conference on Computer Vision. Heidelberg: Springer, 2018: 770-785

[10] Ruan T, Liu T, Huang Z L, et al. Devil in the details: towards accurate single and multiple human parsing[C] //Proceedings of the AAAI Conference on Artificial Intelligence. Palo Alto: AAAI Press, 2019, 33: 4814-4821

[11] Huang X, Wu K Y, Hu G, et al. Multi-class human body parsing with edge-enhancement network[C] //Proceedings of International Conference on Neural Information Processing. Heidelberg: Springer, 2019: 466-477

[12] Chen L C, Papandreou G, Kokkinos I, et al. DeepLab: semantic image segmentation with deep convolutional nets, atrous convolution, and fully connected CRFs[J]. IEEE Transactions on Pattern Analysis and Machine Intelligence, 2018, 40(4): 834-848

[13] Xia F T, Wang P, Chen X J, et al. Joint multi-person pose estimation and semantic part segmentation[C] //Proceedings of the IEEE Conference on Computer Vision and Pattern Recognition. Los Alamitos: IEEE Computer Society Press, 2017: 6769-6778

[14] Nie X C, Feng J S, Yan S C, et al. Mutual learning to adapt for joint human parsing and pose estimation[C] //Proceedings of the European Conference on Computer Vision. Heidelberg: Springer, 2018: 502-517

[15] Liang X D, Gong K, Shen X H, et al. Look into person: joint body parsing \& pose estimation network and a new benchmark[J]. IEEE Transactions on Pattern Analysis and Machine Intelligence, 2019, 41(4): 871-885

[16] Xia F T, Zhu J, Wang P, et al. Pose-guided human parsing by an and/or graph using pose-context features[C] //Proceedings of the 30th AAAI Conference on Artificial Intelligence. Palo Alto: AAAI Press, 2016: 3632-3640

[17] Wang W G, Zhang Z J, Qi S Y, et al. Learning compositional neural information fusion for human parsing[C] //Proceedings of the IEEE International Conference on Computer Vision. Los Alamitos: IEEE Computer Society Press, 2019: 5703-5713

[18] Li J S, Zhao J, Wei Y C, et al. Multiple-human parsing in the wild[OL]. [2020-09-27]. https://arxiv.org/abs/1705.07206.pdf

[19] Ye J W, Feng Z L, Jing Y C, et al. Finer-Net: cascaded human parsing with hierarchical granularity[C] //Proceedings of the IEEE International Conference on Multimedia and Expo. Los Alamitos: IEEE Computer Society Press, 2018: 1-6

[20] Zhao J, Li J S, Cheng Y, et al. Understanding humans in crowded scenes: deep nested adversarial learning and a new benchmark for multi-human parsing[C] //Proceedings of the 26th ACM international conference on Multimedia. New York: ACM Press, 2018: 792-800

[21] Fang H S, Lu G, Fang X, et al. Weakly and semi supervised human body part parsing via pose-guided knowledge transfer[C] //Proceedings of the IEEE Conference on Computer Vi- 
sion and Pattern Recognition. Los Alamitos: IEEE Computer Society Press, 2018: 70-78

[22] Wu Z H, Lin G S, Cai J F. Keypoint based weakly supervised human parsing[J]. Image and Vision Computing, 2019, 91: 103801

[23] Chen Z Y, Liu S, Zhai Y L, et al. Human parsing by weak structural label[J]. Multimedia Tools and Applications, 2018, 77(15): 19795-19809

[24] Zhang Z W, Su C, Zheng L, et al. Correlating edge, pose with parsing[C] //Proceedings of the IEEE Conference on Computer Vision and Pattern Recognition. Los Alamitos: IEEE Computer Society Press, 2020: 8900-8909

[25] Zhang S H, Li R, Dong X, et al. Pose2Seg: detection free human instance segmentation[C] //Proceedings of the IEEE Conference on Computer Vision and Pattern Recognition. Los Alamitos: IEEE Computer Society Press, 2019: 889-898

[26] He K M, Gkioxari G, Dollár P, et al. Mask R-CNN[C]
//Proceedings of the IEEE International Conference on Computer Vision. Los Alamitos: IEEE Computer Society Press, 2017: 2961-2969

[27] Newell A, Yang K, Deng J. Stacked hourglass networks for human pose estimation[C] //Proceedings of European Conference on Computer Vision. Heidelberg: Springer, 2016: 483-499

[28] Yang W, Luo P, Lin L. Clothing co-parsing by joint image segmentation and labeling[C] //Proceedings of the IEEE Conference on Computer Vision and Pattern Recognition. Los Alamitos: IEEE Computer Society Press, 2014: 3182-3189

[29] Russell B C, Torralba A, Murphy K P, et al. LabelMe: a database and web-based tool for image annotation[J]. International Journal of Computer Vision, 2008, 77(1-3): 157-173

[30] Wei S E, Ramakrishna V, Kanade T, et al. Convolutional pose machines $[\mathrm{C}] / /$ Proceedings of the IEEE Conference on Computer Vision and Pattern Recognition. Los Alamitos: IEEE Computer Society Press, 2016: 4724-4732 